\title{
LTI Modell arbiträrer wechselrichtergeführter Inselnetze
}

\author{
LTI Modell of Arbitrary, Voltage Source Inverter Driven, Island Grids
}

M. Jostock, J. Sachau, Universität Luxemburg und C. Tuttas, Universität Kaiserslautern

\begin{abstract}
Der Beitrag beschreibt die Entwicklung eines regelungstechnischen Modells wechselrichtergeführter Inselnetze. Der hier vorgestellte strukturierte Ansatz bildet auf Basis der Zustandsraumdarstellung eines Wechselrichters ein multi-Wechselrichtermodell und verknüpft dieses mit der Strukturinformation der Knoteninzidenzmatrix zu einem geschlossenen Inselnetzmodell. Das Verfahren ermöglicht die Erstellung von Modellen mit beliebig strukturierten Netzen und großer Anzahl unterschiedlicher Wechselrichtermodelle im NS-Netz. Abschließend wird eine Stabilitätsanalyse eines Beispielnetzes präsentiert.
\end{abstract}

This paper presents the development of a control model for inverter driven island grids. The herein presented structured approach creates a multi voltage source inverter model from a single inverter state space model and combines this with the node incidence matrix of a network into a compound model of an island grid. The presented approach allows to analyse arbitrary grid structures and different inverter models and it incorporates a large number of inverters. The model can be particularly used for ohmic-inductive low voltage lines. At the end of the article, a stability analysis of a sample network is presented.

Schlagwörter: Wechselrichter, Inselnetze, Modellierung, Stabilität.

Keywords: Voltage Source Inverter, Island Grids, Modeling, Stability.

\section{Einleitung}

Für den Umbau der elektrischen Energieversorgung auf erneuerbare Energiequellen wird zunehmend die Möglichkeit in Betracht gezogen, Teilnetze im Inselbetrieb zu nutzen. Im Gegensatz zu klassischen Netzen ist in einem solchen Inselnetz kein rotierender Generator vorhanden, sondern die elektrische Energie wird ausschließlich von Wechselrichtern eingespeist. Dass diese nicht leistungsmaximiert alle verfügbare Primärenergie einspeisen können, sondern alle an der koordinierten Frequenz- und Spannungsregelung partizipieren, ist eine Konsequenz der Trennung vom Verbundnetz. Leistung kann nur nach Bedarf eingespeist werden, die Wechselrichter agieren als geregelte Wechselspannungsquellen mit Frequenz-Wirkleistungs-Statik und SpannungsBlindleistungsstatik.

Obwohl der Statikbetrieb schon mehr als 70 Jahre zur Regelung des europäischen Verbundnetzes verwendet wird [13], wurde erst 1997 in einem der ersten europäischen Projekte der EUREC Agentur ein entsprechendes Verfahren zur AC-Kopplung von Wechselrichtern untersucht [10], [5]. 2001 hat Engler nachgewiesen, dass statikbetriebene Wechselrichter angeschlossene Lasten mittels Statiken gleichmäßig versorgen können [4]. Untersuchungen mit mehreren verkoppelten Wechselrichtern folgten [12], jedoch ohne verteilte Netzstrukturen zwischen den Wechselrichtern. Bis dato mangelt es an strukturierten Analysen größerer Netze, in denen eine große Anzahl hochdynamischer Wechselrichter in weit verteilten, ggf. vermaschten Strukturen durch transiente Phasenwinkel interagieren. Von besonderem Interesse sind dabei die Effekte der schnellen Regelung im Millisekundenbereich, ohne die Präsenz stabilisierender, rotierender Generatoren.

Eine Herausforderung bei der Modellierung ist die Berücksichtigung der besonderen Eigenschaften eines Niederspannungsnetzes, vor allem da die klassische 
Netzregelung über Statiken stets von induktiven Leitungen ausgehen, die im Niederspannungsnetz mitnichten angenommen werden können.

Neuere Ansätze finden sich z.B. in [11], [3], [14] und [6]. Die Modelle basieren entweder auf der expliziten Formulierung der Differentialgleichungssysteme, die für jede Netzstruktur neu hergeleitet werden müssen und somit nur für sehr kleine Netze berechenbar waren, oder sie basieren auf dem Kuramotomodell verkoppelter Oszillatoren, die für Wechselrichtersysteme nur bedingt anwendbar sind.

Aus regelungstechnischer Sicht ist ein wechselrichtergeführtes Inselnetz ein verteiltes Multi-Aktor-System, bei dem verschiedene Aktuatoren auf dieselben Größen (Frequenz und Spannung) an verschiedenen Punkten Einfluss nehmen.

Aus energietechnischer Sicht handelt es sich um ein Netz, bei dem die Frequenz nicht auf $50 \mathrm{~Hz}$ fixiert ist, sondern in Abhängigkeit der Lastsituation schwankt.

Der vorliegende Text stellt ein lineares, zeitinvariantes Modell vor, welches weit verteilte, arbiträre Netzstrukturen umfasst, ohmsch-induktive Leitungen berücksichtigt und beliebig viele Wechselrichter mit ihrem jeweils unterschiedlichen dynamischen Verhalten einbezieht, um Aussagen über die Stabilität eines solchen Netzes treffen zu können.

Im Anschluss wird die Anwendbarkeit an Beispielen demonstriert.

\section{Ansatz}

Die Wechselrichter sind Teil der Gesamtsystemdynamik und beeinflussen sich im Inselnetz gegenseitig. Im Inselnetzbetrieb operieren sie mit einer Frequenz-Wirkleistungs-Statik $\mathrm{f}(\mathrm{P})$ und SpannungsBlindleistungs-Statik $\mathrm{u}(\mathrm{Q})$ und werden als Wechselspannungsquellen betrachtet. Sie stellen Spannung und Phasenwinkel an ihren Klemmen anhand des an aktuell auftretenden Lastflusses (Wirkleistung $P$ und Blindleistung $Q$ ) ein. Arbeitet das Netz stabil, dann werden sich nach einer Laständerung, im Anschluss an eine transiente Phase, die Lastflüsse auf alle Wechselrichter gleichmäßig, gemäß ihrer Statik aufteilen, ohne definierte Randbedingungen während der transienten Phase zu verletzen.

Der Schlüssel zur Modellierung als Mehrgrößensystem (MIMO - multiple input multiple output) liegt in der Aufteilung des Gesamtsystems in aktive und passive Komponenten. Das Modell unterscheidet deshalb jene Netzknoten an denen aktive Wechselrichter angeschlossen sind, von den Netzknoten, an denen Verbraucher angeschlossen sein können. Eine Randbedingung ist, dass die jeweiligen Knotenmengen disjunkt sind. Die Wechselrichter werden als Teilsysteme modelliert, die über die Impedanzen des Netzes verkoppelt sind. Systemeingänge sind die Lastflüsse $P_{\text {Load } i}$ und $Q_{\text {Load } i}$ an diesen passiven Knoten. Systemausgänge sind die sich an den passiven Knoten einstellende Spannungsamplitude $u_{i}$ und Phasenwinkel $\delta_{i}$.

Da das sich ergebende Gesamtmodell genauso viele $\mathrm{Zu}$ standsgrößen wie die Summe der Teilsysteme enthält, ergibt sich kein überbestimmtes System von Differentialgleichungen (DGL) mit Nebenbedingungen, sondern ein geschlossen lösbares DGL-System.

Jeder der $w$ Wechselrichter verändert seine Stellgrößen Spannungsamplitude $u_{i}$ und Frequenz $f_{i}$ (und damit den Phasenwinkel $\delta_{i}$ ) aufgrund der regelungstechnischen Eingangsgrößen Wirkleistung $P_{i}$ und Blindleistung $Q_{i}$ an ihren Klemmen. Sie beeinflussen so die Wirk- und Blindleistungsströme im passiven Netzwerk.

Da die Dynamik des Leistungsflusses in den Netzleitungen von physikalischen Gesetzen bestimmt wird, laufen diese wesentlich schneller ab, als die Regelalgorithmen der Wechselrichter. Das Prinzip der singulären Perturbation erlaubt daher eine getrennte Betrachtung der beiden Teilsysteme. Die logische Trennung in aktives Wechselrichtersystem und passives Netz ist in Abbildung 1 dargestellt.

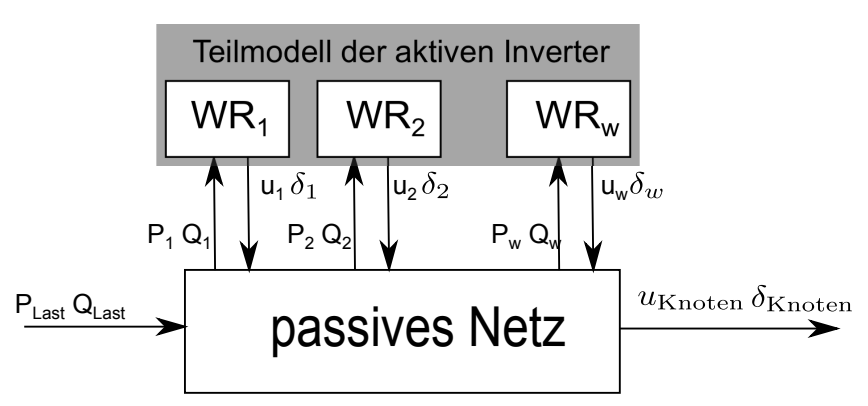

Bild 1: Gesamtsystem mit logischer Trennung zwischen Netzwerk-Modell und Mulit-Wechselrichter-Modell

Anhand der an den Wechselrichterklemmen eingestellten Spannungswinkel $\left(\delta_{i}, \ldots, \delta_{w}\right)$ und Spannungsamplituden $\left(u_{1}, \ldots, u_{w}\right)$ berechnet das Netz-Teilmodell die Leistungsflüsse der Wirkleistungen $\left(P_{1}, \ldots, P_{w}\right)$ und Blindleistungen $\left(Q_{1}, \ldots, Q_{w}\right)$, die an den Wechselrichterknoten ins Netz fließen.

Betrachtet werden soll sowohl die Ein/Ausgangsstabilität als auch die Zustandsstabilität des Gesamtsystems. Dies bedeutet, dass die Ableitung von z.B. Pol-Nullstellen-Diagrammen möglich sein muss, um die Stabilität, zumindest in einem gegebenen Arbeitspunkt, in Abhängigkeit von beeinflussbaren Wechselrichterparametern zu beurteilen. Strukturvarianz wird nicht betrachtet, d.h. Veränderungen der Lastverhältnisse werden als Variation von Eingangsvariablen interpretiert und nicht als Strukturveränderung. ${ }^{1}$

1 Im Gegensatz zur Analyse klassischer Energieverteilnetze, wo Lastflüsse i.A. in Impedanzen umgerechnet werden, 
Für unterschiedliche Strukturen müssen jeweils neue Netzmodelle aus der jeweiligen Strukturbeschreibung generiert werden. Zur Darstellung von z.B. PolNullstellen-Diagrammen wird eine lineare Beschreibung des Systems benötigt, d.h. es muss das Modell eines LTISystems (linear, zeitinvariant) entwickelt werden.

\section{Wechselrichtermodell im Zustandsraum}

Die im System betrachteten Wechselrichter können für verschiedene Statiken untersucht werden. Die Beschreibung des Wechselrichters erfolgt in der Zustandsraumdarstellung.

\subsection{Einzelwechselrichter}

Da die Variable $u$ für die Spannungsamplitude verwendet wird, wird der Eingangsvektor hier mit $\mathfrak{s}$ bezeichnet, in Anlehnung an die Scheinleistung S . Die Zustandsraumdarstellung. Der Ausgangsvektor wird mit $\nu$ bezeichnet, da mit $y$ eine Zweigadmittanz bezeichnet wird. Das Modell wird beschrieben mit

$$
\begin{aligned}
& \dot{x}=\mathcal{A} x+\mathcal{B} \mathfrak{s} \\
& \nu=\mathcal{C} x+\mathcal{D} \mathfrak{s}
\end{aligned}
$$

Ausgehend vom Wechselrichterverhalten als masseloser Synchrongenerator (siehe auch [4]), kann das regelungstechnische Modell im Arbeitspunk $\left[u_{0}, f_{0}\right]$ in Abbildung 2 dargestellt werden:

Die Eingangsgröße $\overrightarrow{\mathfrak{s}}$ des Wechselrichters besteht aus der Wirkleistung $\mathrm{P}$ und der Blindleistung Q. Der Ausgangsvektor $\vec{\nu}$ des Wechselrichters besteht aus dem Winkel $\delta$ und der Spannung u. Da der Wechselrichter bei Betrieb mit einer $\mathrm{f}(\mathrm{P})$-Statik seine eigene Einspeisefrequenz ändert, wird die Winkelgeschwindigkeit ${ }^{2}$ $\dot{\varphi}_{\text {VsI }}$ der Wechselrichterspannungals zusätzliche Ausgangsgröße nach außen geführt.

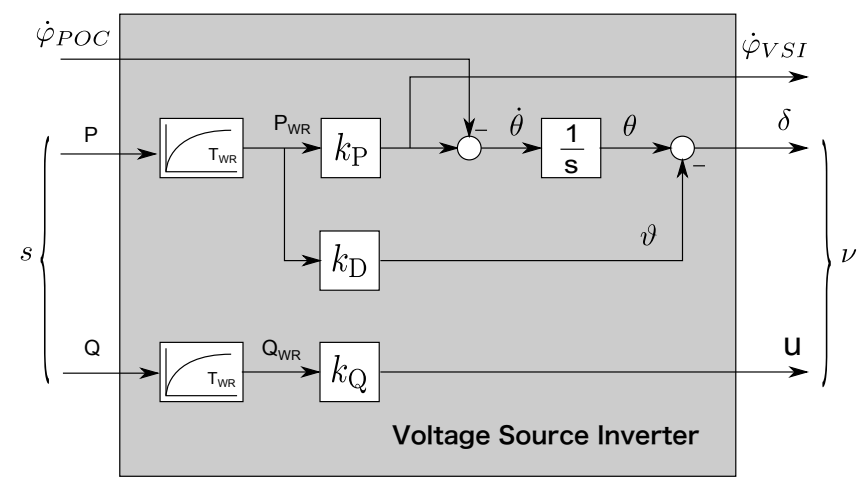

Bild 2: Wechselrichter im Arbeitspunkt $\left[f_{0}, u_{0}\right]$

sind in diesem Model die Zweigleistungsflüsse explizit Systemvariablen.

2 Genauer: $\dot{\varphi}_{\text {VSI }}$ ist die Abweichung der Winkelgeschwindigkeit von der Nennfrequenz $f_{0}$ im Arbeitspunkt.
Die Leistungen werden vom Wechselrichter an seinen Klemmen gemessen. Am Eingang liegt ein VZ1Glied, das mit der Zeitkonstanten $T_{\mathrm{WR}}$ modelliert wird. Alle Verzögerungen des Wechselrichters werden in dieser Zeitkonstanten zusammengefasst: Messwerterfassung, Filterung, Leistungsberechnung, Raumzeigermodulation (PWM) und Stromregelung. Weiters kann diese Zeitkonstante künstlich vergrößert werden, um eine aufgeprägte Trägheit zu simulieren. Die Leistungen, die dann in die Statik eingehen lauten $P_{\mathrm{WR}}$ und $Q_{\mathrm{WR}}$. Diese werden über die Statiken $k_{P}$ bzw. $k_{Q}$ in die entsprechende Frequenz- bzw. Spannungsänderung umgerechnet. Die Faktoren enthalten die jeweilige Statik (droop) bezogen auf die Nennleistungen $P_{n}$ und $Q_{n}$ :

$$
\begin{aligned}
& k_{P}=-\frac{\text { droop }_{\mathrm{P}}}{P_{n}}, \\
& k_{Q}=-\frac{\text { droop }_{\mathrm{Q}}}{Q_{n}},
\end{aligned}
$$

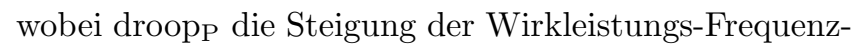
Statik ist (z.B. droop $p_{P}=2 \%$ ) und droop ${ }_{Q}$ die Steigung der Blindleistungs-Spannungs-Statik (z.B. droop $_{\mathrm{Q}}=$ 4\%). Im Blindleistungszweig wird die Spannung als Ausgangswert bestimmt. Im Wirkleistungspfad führt eine von der aktuellen Winkelgeschwindigkeit im Anschlusspunkt $\dot{\varphi}_{\text {POC }}$ abweichende Einspeisefrequenz $\dot{\varphi}_{\text {VSI }} \mathrm{zu}$ einem Winkeldrift. Dieser Drift wird durch die Integration der Winkelgeschwindigkeitsdifferenz berechnet und ergibt den Driftwinkel $\theta$. Zu dem Driftwinkel kann noch eine direkt von der Wirkleistung $P_{\mathrm{WR}}$ abhängige Phasenvorsteuerung addiert werden die sich als Dämpfung mit dem Faktor $k_{D}$ bemerkbar macht. Daraus entsteht der Ausgangsphasenwinkel $\delta$.

\section{Anmerkung}

$\dot{\varphi}_{\mathrm{POC}}$ kann als Sollwertvorgabe für den Wirkleistungszweig gesehen werden. Solange eine Abweichung $\dot{\theta}=$ $\dot{\varphi}_{\mathrm{POC}}-\dot{\varphi}_{\mathrm{VSI}}$ existiert, wird der Wechselrichter seinen Phasenwinkel $\delta$ variieren. Dadurch ändern sich die Leistungsflüsse in den anderen Wechselrichtern, die auf Grund ihrer $f(P)$-Statik ihre eigene Einspeisefrequenz anpassen. Diese wirkt dann über $\dot{\varphi}_{\mathrm{POC}}$ auf alle anderen Wechselrichter zurück. Nach einer transienten Zeitspanne wird bei allen Wechselrichtern $\dot{\varphi}_{\mathrm{VSI}}$ mit $\dot{\varphi}_{\mathrm{POC}}$ übereinstimmen und die Phasenwinkel bleiben stabil.

Für die Leistungsflussberechnung ist nicht die Frequenz relevant, sondern der Phasenwinkel. Dies erklärt sich damit, dass ein Wechselrichter zwar bei einer $\mathrm{f}(\mathrm{P})$-Statik die Frequenz seiner Ausgangsspannung anpasst, dies jedoch zu einem Phasendrift gegenüber dem über eine Admittanz verbundenen nächsten Netzknoten und damit zu den anderen Wechselrichtern führt, was wiederum deren Leistungsfluss beeinflusst. Dieser Phasendrift lässt sich direkt durch den am Anschlusspunkt anliegenden Spannungs-Phasenwinkel $\delta$ ausdrücken.

Für das in Abb. 2 dargestellte Strukturbild können die Differentialgleichungen aufgestellt werden. Für das 
Verzögerungsglied 1. Ordnung (VZ1) im Wirkleistungszweig gilt:

$$
T_{\mathrm{WR}} \dot{P}_{\mathrm{WR}}+P_{\mathrm{WR}}=P
$$

und daraus folgt

$$
\dot{P}_{\mathrm{WR}}=-\frac{1}{T_{\mathrm{WR}}} P_{\mathrm{WR}}+\frac{1}{T_{\mathrm{WR}}} P
$$

und entsprechend für den Blindleistungszweig

$$
\dot{Q}_{\mathrm{WR}}=-\frac{1}{T_{\mathrm{WR}}} Q_{\mathrm{WR}}+\frac{1}{T_{\mathrm{WR}}} Q .
$$

Für den Winkel $\theta$ gilt

$$
\dot{\theta}=k_{\mathrm{P}} \cdot P_{\mathrm{WR}}-\dot{\varphi}_{\mathrm{POC}}
$$

und der Ausgangswinkel $\varphi$ setzt sich zusammen aus

$$
\delta=\theta-k_{D} P_{\mathrm{WR}}
$$

Der Zustandsvektor $\vec{x}$ wird definiert als

$$
\vec{x}=\left[\begin{array}{c}
P_{\mathrm{WR}} \\
Q_{\mathrm{WR}} \\
\theta
\end{array}\right] .
$$

Damit ergibt sich aus den Gleichungen 1 bis 4 für die Zustandsraumdarstellung des in Abbildung 2 dargestellten Strukturbildes

$$
\begin{aligned}
& \frac{d}{d t}\left[\begin{array}{c}
P_{\mathrm{WR}} \\
Q_{\mathrm{WR}} \\
\theta
\end{array}\right]=\left[\begin{array}{ccc}
-\frac{1}{T_{\mathrm{WR}}} & 0 & 0 \\
0 & -\frac{1}{T_{\mathrm{WR}}} & 0 \\
k_{\mathrm{P}} & 0 & 0
\end{array}\right]\left[\begin{array}{c}
P_{\mathrm{WR}} \\
Q_{\mathrm{WR}} \\
\theta
\end{array}\right] \\
& +\left[\begin{array}{ccc}
\frac{1}{T_{\mathrm{WR}}} & 0 & 0 \\
0 & \frac{1}{T_{\mathrm{WR}}} & 0 \\
0 & 0 & -1
\end{array}\right]\left[\begin{array}{c}
P \\
Q \\
\dot{\varphi}_{\mathrm{POC}}
\end{array}\right] \\
& {\left[\begin{array}{c}
\delta \\
u \\
\dot{\varphi}_{\mathrm{VSI}}
\end{array}\right]=\left[\begin{array}{ccc}
-k_{D} & 0 & 1 \\
0 & k_{Q} & 0 \\
k_{P} & 0 & 0
\end{array}\right]\left[\begin{array}{c}
P_{\mathrm{WR}} \\
Q_{\mathrm{WR}} \\
\theta
\end{array}\right]}
\end{aligned}
$$

Die Zustandsraumdarstellung (Gl. 5) lässt sich aufteilen: Die leistungsbezogenen Größen werden getrennt von den frequenzbezogenen Größen und dadurch ergibt sich vertikale Aufspaltung der Eingangsmatrix $\mathcal{B}$ und eine horizontale Aufspaltung der Ausgangsmatrix $\mathcal{C}$. Der Eingangsvektor für einen einzelnen Wechselrichter setzt sich zusammen aus $\overrightarrow{\mathfrak{s}}=[P Q]^{T}$ und $\dot{\varphi}_{\text {POC }}$, der Ausgangsvektor setzt sich zusammen aus $\vec{\nu}=[\delta u]^{T}$ und $\dot{\varphi}_{\text {VSI }}$.

$$
\begin{aligned}
\dot{\vec{x}} & =\mathcal{A} \vec{x}+\left[\begin{array}{ll}
\mathcal{B}_{s} \mathcal{B}_{f}
\end{array}\right]\left[\begin{array}{c}
\overrightarrow{\mathfrak{s}} \\
\dot{\varphi}_{\mathrm{POC}}
\end{array}\right] \\
{\left[\begin{array}{c}
\vec{\nu} \\
\dot{\varphi}_{\mathrm{VSI}}
\end{array}\right] } & =\left[\begin{array}{l}
\mathcal{C}_{\nu} \\
\mathcal{C}_{f}
\end{array}\right] \vec{x}
\end{aligned}
$$

Damit ergibt sich folgende Zustandsraumdarstellung für den Wechselrichter.

$$
\begin{aligned}
\dot{\vec{x}} & =\mathcal{A} \vec{x}+\mathcal{B}_{s} \overrightarrow{\mathfrak{s}}+\mathcal{B}_{f} \dot{\varphi}_{\mathrm{POC}} \\
\vec{\nu} & =\mathcal{C}_{\nu} \vec{x} \\
\dot{\varphi}_{\mathrm{VSI}} & =\mathcal{C}_{f} \vec{x}
\end{aligned}
$$

mit

$$
\begin{aligned}
& \mathcal{B}_{s}=\left[\begin{array}{cc}
\frac{1}{T_{\mathrm{WR}}} & 0 \\
0 & \frac{1}{T_{\mathrm{WR}}} \\
0 & 0
\end{array}\right] \\
& \mathcal{B}_{f}=\left[\begin{array}{c}
0 \\
0 \\
-1
\end{array}\right], \\
& \mathcal{C}_{\nu}=\left[\begin{array}{ccc}
-k_{D} & 0 & 1 \\
0 & k_{Q} & 0
\end{array}\right], \\
& \mathcal{C}_{f}=\left[\begin{array}{llll}
k_{P} & 0 & 0
\end{array}\right] .
\end{aligned}
$$

Die Matrix $\mathcal{D}$ ist eine Nullmatrix, da hier kein direkter Durchgriff des Eingangs auf den Ausgang besteht. Das Strukturbild der Zustandsraumdarstellung zeigt Abb. 3.

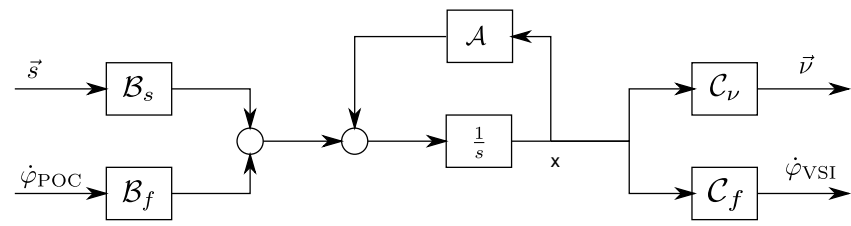

Bild 3: Zustandsraummodell des Wechselrichters

\subsection{Multi-Wechselrichter Modell}

Werden mehrere Wechselrichter im Gesamtmodell zu einem einzigen Block zusammengefasst, so ergibt sich die Zustandsraumbeschreibung aller Wechselrichter in Form von Blockdiagonalmatrizen.

$$
\begin{aligned}
& \frac{d}{d t}\left[\begin{array}{c}
\vec{x}_{1} \\
\vec{x}_{2} \\
\vdots \\
\vec{x}_{n}
\end{array}\right]=\left[\begin{array}{cccc}
\mathcal{A}_{1} & & \cdots & 0 \\
& \mathcal{A}_{2} & & \vdots \\
\vdots & & \ddots & \\
0 & \cdots & & \mathcal{A}_{n}
\end{array}\right] \cdot\left[\begin{array}{c}
\vec{x}_{1} \\
\vec{x}_{2} \\
\vdots \\
\vec{x}_{n}
\end{array}\right] \\
& +\left[\begin{array}{cccc}
\mathcal{B}_{s 1} & & \cdots & 0 \\
& B_{s 2} & & \vdots \\
\vdots & & \ddots & \\
0 & \cdots & & B_{s n}
\end{array}\right] \cdot\left[\begin{array}{c}
\overrightarrow{\mathfrak{s}}_{1} \\
\overrightarrow{\mathfrak{s}}_{2} \\
\vdots \\
\overrightarrow{\mathfrak{s}}_{n}
\end{array}\right] \\
& +\left[\begin{array}{cccc}
\mathcal{B}_{f 1} & & \cdots & 0 \\
& B_{f 2} & & \vdots \\
\vdots & & \ddots & \\
0 & \cdots & & B_{f n}
\end{array}\right] \cdot\left[\begin{array}{c}
\dot{\varphi}_{\mathrm{POC} 1} \\
\dot{\varphi}_{\mathrm{POC} 2} \\
\vdots \\
\dot{\varphi}_{\mathrm{POCn}}
\end{array}\right]
\end{aligned}
$$




$$
\begin{gathered}
{\left[\begin{array}{c}
\vec{\nu}_{1} \\
\vec{\nu}_{2} \\
\vdots \\
\vec{\nu}_{n}
\end{array}\right]=\left[\begin{array}{cccc}
\mathcal{C}_{\nu 1} & & \cdots & 0 \\
& \mathcal{C}_{\nu 2} & & \vdots \\
\vdots & & \ddots & \\
0 & \cdots & & \mathcal{C}_{\nu n}
\end{array}\right] \cdot\left[\begin{array}{c}
\vec{x}_{1} \\
\vec{x}_{2} \\
\vdots \\
\vec{x}_{n}
\end{array}\right]} \\
{\left[\begin{array}{c}
\dot{\varphi}_{\text {VSI1 }} \\
\dot{\varphi}_{\text {VSI2 }} \\
\vdots \\
\dot{\varphi}_{\text {VSIn }}
\end{array}\right]=\left[\begin{array}{cccc}
\mathcal{C}_{f 1} & & \cdots & 0 \\
& \mathcal{C}_{f 2} & & \vdots \\
\vdots & & \ddots & \\
0 & \cdots & & \mathcal{C}_{f n}
\end{array}\right] \cdot\left[\begin{array}{c}
\vec{x}_{1} \\
\vec{x}_{2} \\
\vdots \\
\vec{x}_{n}
\end{array}\right]}
\end{gathered}
$$

Die Eingangs- Ausgangs- und Zustandsvektoren können in der Multi-Wechselrichter Darstellung zusammengefasst werden zu:

$$
\begin{aligned}
\vec{s} & =\left[\begin{array}{lllll}
\overrightarrow{\mathfrak{s}}_{1}^{T} & \overrightarrow{\mathfrak{s}}_{2}^{T} & \overrightarrow{\mathfrak{s}}_{3}^{T} & \cdots & \overrightarrow{\mathfrak{s}}_{n}^{T}
\end{array}\right]^{T}, \\
\vec{\nu} & =\left[\begin{array}{lllll}
\vec{\nu}_{1}^{T} & \vec{\nu}_{2}^{T} & \vec{\nu}_{3}^{T} & \cdots & \vec{\nu}_{n}^{T}
\end{array}\right]^{T}, \\
\vec{x} & =\left[\begin{array}{lllll}
\vec{x}_{1}^{T} & \vec{x}_{2}^{T} & \vec{x}_{3}^{T} & \cdots & \vec{x}_{n}^{T}
\end{array}\right]^{T} .
\end{aligned}
$$

Die Berechnung der Anschlusspunktwinkelgeschwindigkeiten $\dot{\varphi}_{\text {POCi }}$ aus den verschiedenen Wechselrichterphasengeschwindigkeiten $\dot{\varphi}_{\text {VSIi }}$ kann innerhalb der BlockModellierung zusammengefasst werden, so dass die Wechselrichter mit dem Netzmodell nur $\vec{s}$ und $\vec{\nu}$ austauschen, d.h. diese sind die einzigen Eingangs- bzw. Ausgangsgrößen für die Multi-Wechselrichter Beschreibung.

Da ein LTI-Modell zur Stabilitätsbetrachtung benötigt wird, muss ein plausibler Ansatz für eine lineare Berechnung der Phasengeschwindigkeiten an jedem Anschlusspunkt eines Wechselrichters gefunden werden. Ändert ein Wechselrichter, z.B. auf Grund eines räumlich nahen Lastsprungs seine Einspeisefrequenz, muss dies eine Auswirkung auf die gesamte Netzfrequenz haben, die sich auf Grund der Statiken nach einer transienten Phase auf einen neuen Wert einstellt. Diese Berechnung der Anschlusspunktfrequenzen (Phasengeschwindigkeiten $\left.\overrightarrow{\dot{\varphi}}_{\text {POC }}\right)$ wird über die Matrix $\mathcal{M}_{f}$ durchgeführt:

$$
\overrightarrow{\dot{\varphi}}_{\mathrm{POC}}=\mathcal{M}_{f} \overrightarrow{\dot{\varphi}}_{\mathrm{VSI}}
$$

Osika hat diesen Aspekt in [12] als arithmetisches Mittel aller Einspeisefrequenzen modelliert.

In Abbildung 4 ist erkennbar, dass der Zweig über $\mathcal{A}$ und der Zweig über $\mathcal{M}_{f}$ parallel liegen. Diese beiden Zweige können deshalb zu einer einzigen Matrix

$$
\mathcal{A}^{\prime}=\mathcal{A}+\mathcal{B}_{f} \mathcal{M}_{f} \mathcal{C}_{f}
$$

zusammengefasst werden. Damit erhält man das in Abbildung 4 dargestellte Gesamtmodell für alle Wechselrichter im Netzwerk. Dieses Modell wird im folgenden Teil in die Modellierung des Gesamtsystems integriert.

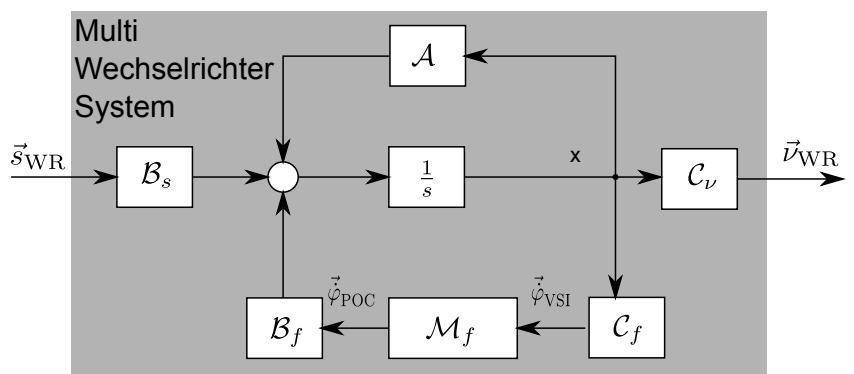

Bild 4: Alle Wechselrichter des Netzes in einem Block im Zustandsraum

\section{Leistungsgleichungen}

Die Systemmodellierung des passiven Netzmodells beginnt mit der Berechnung der übertragenen Leistung zwischen zwei Netzknoten.

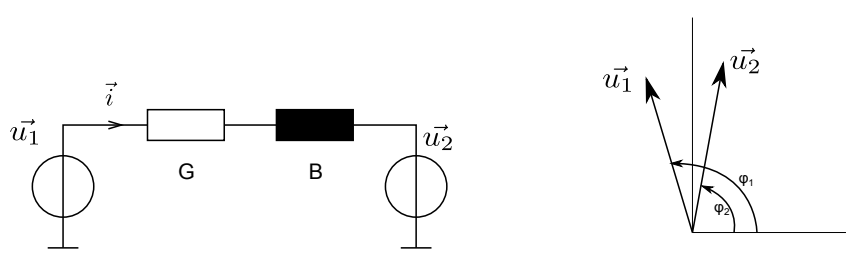

Bild 5: Lastfluss zwischen zwei Spannungsknoten

Die Leitwerte berechnen sich aus dem Widerstandswert $\mathrm{R}$ und der Induktivität L zu

$$
G=\frac{R}{R^{2}+(\omega L)^{2}}
$$

und

$$
B=\frac{-\omega L}{R^{2}+(\omega L)^{2}}
$$

Um den Leistungsfluss von Knoten 1 nach Knoten 2 berechnen zu können, wird erst der Strom i ermittelt. Mit $u_{1}(t)=u_{1} \cdot e^{j \omega t+\varphi_{1}}$ und $u_{2}(t)=u_{2} \cdot e^{j \omega t+\varphi_{2}}$ folgt mit gleicher Phasenreferenz für i und $u_{1}$ :

$$
\begin{aligned}
i= & (G+j B) \cdot\left(u_{1}-u_{2} e^{\mathrm{j}\left(\varphi_{2}-\varphi_{1}\right)}\right) \cdot e^{j \omega t} \\
= & (G+j B) \cdot\left[u_{1}-\left(u_{2} \cos \left(\varphi_{2}-\varphi_{1}\right)+j \cdot u_{2} \sin \left(\varphi_{2}-\varphi_{1}\right)\right)\right] \cdot e^{j \omega t} \\
= & (G+j B) \cdot\left[u_{1}-u_{2} \cos \left(\varphi_{1}-\varphi_{2}\right)+j \cdot u_{2} \sin \left(\varphi_{1}-\varphi_{2}\right)\right] \cdot e^{j \omega t} \\
= & {\left[-B u_{2} \sin \left(\varphi_{1}-\varphi_{2}\right)+G\left(u_{1}-u_{2} \cos \left(\varphi_{1}-\varphi_{2}\right)\right)\right.} \\
& \left.+j \cdot\left(G u_{2} \sin \left(\varphi_{1}-\varphi_{2}\right)+B\left(u_{1}-u_{2} \cos \left(\varphi_{1}-\varphi_{2}\right)\right)\right)\right] \cdot e^{j \omega t}
\end{aligned}
$$

und daraus folgt der Leistungsfluss aus Knoten 1 nach Knoten 2

$$
\begin{aligned}
P+j Q= & u_{1} \cdot i^{\star} \\
= & -B u_{1} u_{2} \sin \left(\varphi_{1}-\varphi_{2}\right)+G\left(u_{1}^{2}-u_{1} u_{2} \cos \left(\varphi_{1}-\varphi_{2}\right)\right) \\
& +j \cdot\left(-G u_{1} u_{2} \sin \left(\varphi_{1}-\varphi_{2}\right)-B\left(u_{1}^{2}-u_{1} u_{2} \cos \left(\varphi_{1}-\varphi_{2}\right)\right)\right)
\end{aligned}
$$

bzw. in Matrizendarstellung

$$
\left[\begin{array}{l}
P \\
Q
\end{array}\right]=\left[\begin{array}{cc}
-B & G \\
-G & -B
\end{array}\right] \cdot\left[\begin{array}{c}
u_{1} u_{2} \sin \left(\varphi_{1}-\varphi_{2}\right) \\
u_{1}^{2}-u_{1} u_{2} \cos \left(\varphi_{1}-\varphi_{2}\right)
\end{array}\right]
$$


Gleichung 15 ist der zentrale Ausgangspunkt für die Modellierung des linearisierten Leistungsflusses im Wechselrichternetzwerk.

\subsection{Generelle Konventionen}

Der Leistungsvektor mit der Wirk- und Blindleistung über einen Zweig $\mathrm{k}$ wird mit $\vec{s}_{k}$ zusammengefasst:

$$
\vec{s}_{k}=\left[\begin{array}{c}
P_{k} \\
Q_{k}
\end{array}\right]
$$

Die Leitwertmatrix eines Zweiges $\mathrm{k}$ mit einem Wirk- und einem Blindleitwert wird mit $y_{k}$ bezeichnet:

$$
y_{k}=\left[\begin{array}{cc}
-B_{k} & G_{k} \\
-G_{k} & -B_{k}
\end{array}\right]
$$

Der Vektor von Spannungen und Winkel zwischen zwei Knoten i und j wird mit dem griechischen $\vec{\chi}$ bezeichnet. Im nicht-linearen Fall also

$$
\overrightarrow{\chi_{i j}}=\left[\begin{array}{c}
u_{i} u_{j} \sin \left(\varphi_{i}-\varphi_{j}\right) \\
u_{i}^{2}-u_{i} u_{j} \cos \left(\varphi_{i}-\varphi_{j}\right)
\end{array}\right]
$$

Damit ergibt sich die Leistungsgleichung für den Energiefluss von Knoten i über Zweig k nach Knoten j zu

$$
\vec{s}_{k}=y_{k} \cdot \vec{\chi}_{i j}
$$

\subsection{Linearisierung}

Da ein LTI-Modell benötigt wird, werden die Leistungsgleichungen nach den aus der Energietechnik bekannten Methoden linearisiert. Unter der Annahme, dass nur kleine Winkeldifferenzen zwischen den Netzknoten vorliegen, und die Spannungen i.A. in der Nähe der Referenzspannung $u_{0}=1.0 p$.u. liegen, hängt die Wirkleistung maßgeblich von der Winkeldifferenz ab und die Blindleistung von der Spannungsdifferenz. Es gilt

$$
\vec{\chi}_{i j} \approx\left[\begin{array}{l}
\varphi_{i}-\varphi_{j} \\
u_{i}-u_{j}
\end{array}\right]
$$

Es wurde durch die Linearisierung ${ }^{3}$ also ein Übergang zum Kleinsignalmodell vollzogen. Im Besonderen gilt als Konsequenz dieser Linearisierung: $\vec{\chi}_{i j}=-\vec{\chi}_{j i}$. Die Leitungen werden also als verlustlos betrachtet.

Für die Berechnung des Leistungsvektors $\vec{s}_{k}$ folgt daraus

$$
\begin{aligned}
& \vec{s}_{k}=\mathbf{y}_{k} \cdot \vec{\chi}_{i j} \\
& \vec{s}_{k}=\mathbf{y}_{k} \cdot\left(\left[\begin{array}{l}
\varphi_{i} \\
u_{i}
\end{array}\right]-\left[\begin{array}{l}
\varphi_{j} \\
u_{j}
\end{array}\right]\right) \\
& \vec{s}_{k}=\mathbf{y}_{k} \cdot\left(\vec{\nu}_{i}-\vec{\nu}_{j}\right)
\end{aligned}
$$

3 Engler kommt in [4] zu ähnlichen Schlüssen in seiner Modellierung eines Wechselrichtersystems, verwendet jedoch für seine Annahmen rein induktiv gekoppelte Wechselrichter. Dadurch wird die Admittanzmatrix y zu einer Diagonalmatrix, was zu einer Entkopplung von $P$ und $Q$ führt und die Aufspaltung in zwei unabhängige mathematische Probleme gestattet. $\operatorname{mit} \vec{\nu}_{i}=\left[\begin{array}{c}\varphi_{i} \\ u_{i}\end{array}\right]$.

Durch die Zerlegung von $\vec{\chi}_{i j}$ in zwei linear unabhängige Knotenspannungsvektoren $\vec{\nu}_{i}$ und $\vec{\nu}_{j}$, kann der Leistungsvektor $\vec{s}_{k}$ für bekannte Knotenspannungen in jedem Zweig des Netzwerkes berechnet werden. Wichtiger noch, kann auf Grund der linearen Gleichung 17 aus $\vec{s}_{k}$, $\vec{\nu}_{i}$ oder $\vec{\nu}_{j}$ bei zwei bekannten Größen die dritte Größe berechnet werden.

Die Anwendbarkeit der Linearisierung von AC Leitungen unter Vernachlässigung von Leitungsverlusten wurde in diversen Arbeiten untersucht, z.B. in [2] und [1].

\section{Statisches Netzmodell}

Ausgehend von der in Gl. 17 dargestellten Vereinfachung der Beziehungen zwischen Zweigleistungen und Knotenspannungen bzw. -winkel, wird das linearisierte Netzmodell des gesamten Inselnetzes entwickelt.

\subsection{Inzidenzmatrix}

Gegeben sei folgendes Beispiel mit dem Netzwerk aus Abbildung 6 in dem die $t$ Zweige (twigs) nummeriert und die $n$ Knoten (nodes) buchstabiert sind ${ }^{4}$.

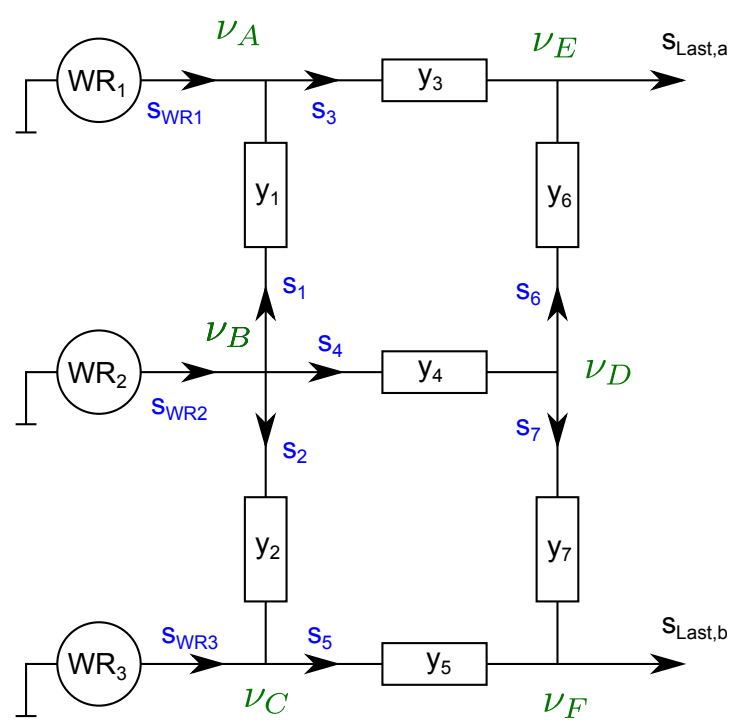

Bild 6: Beispielnetz zur Modellbildung

Aus dem Netzwerk kann die $n \times t$ Inzidenzmatrix $\mathcal{K}$ ermittelt werden, wobei ein zu einem Knoten gerichteter Zweig mit 1 und von einem Knoten weg gerichteter Zweig mit -1 eingetragen wird. Die Richtung entspricht

4 Die Notation der Knotenbuchstaben und Zweigzahlen wird in diesem Dokument dauerhaft beibehalten 
dem positiven Leistungsfluss. Aus Abbildung 6 ergibt sich folgende Inzidenzmatrix $\mathcal{K}$ :

$$
\begin{aligned}
& \text { tZweige } \\
& \begin{array}{lllllll}
1 & 2 & 3 & 4 & 5 & 6 & 7
\end{array}
\end{aligned}
$$

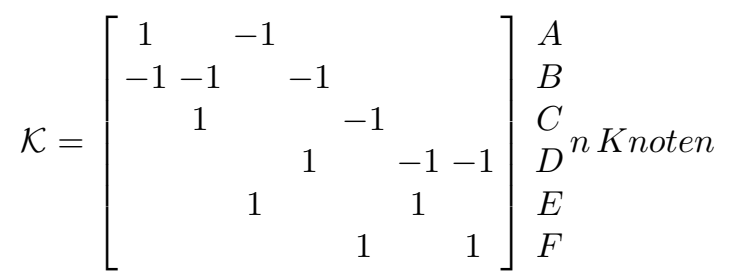

- Die Knoten A,B und C sind Knoten an denen Wechselrichter angeschlossen sind und diese Zeilen sind in $\mathcal{K}$ nach oben sortiert. Die Position der Wechselrichter gehört zur Systemstruktur und ist invariant.

- Alle verbleibenden Knoten D, E und F sind potentielle Lastknoten, an ihnen können Verbraucherlasten abfließen. Ort und Betrag der Lastflüsse gehören zum Eingangslastvektor $\vec{s}_{L}$. Diese Knoten stehen in $\mathcal{K}$ unten.

- Die Menge der Wechselrichterknoten und der Netzknoten ist in diesem Modell disjunkt! D.h. Lastabflüsse direkt am Wechselrichter werden nicht betrachtet. $^{5}$

Somit lässt sich die Matrix $\mathcal{K}$ horizontal aufspalten in eine Submatrix $\mathcal{K}_{W R}$ mit $w$ Zeilen für die Wechselrichterknoten und $\mathcal{K}_{N}$ mit $l$ Zeilen (load) für die Netzkno$\operatorname{ten}^{6}$ :

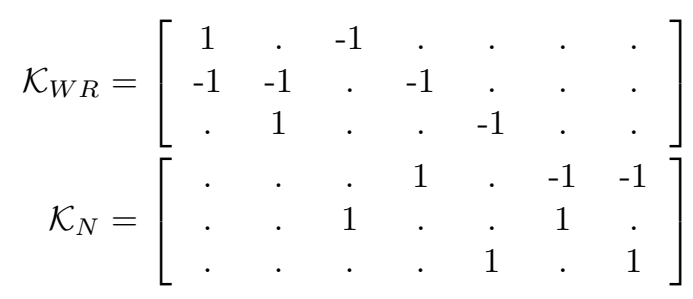

Wird die Inzidenzmatrix $\mathcal{K}$ multipliziert mit dem Lei-

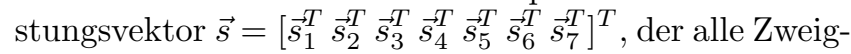
leistungen enthält, so ergibt sich zusammen mit den einfließenden Leistungen der Wechselrichter $\vec{s}_{\mathrm{WR}}$ und den abfließenden Leistungen der Lasten $\vec{s}_{L}$ ein Gleichungssatz, der den Kirchhoffschen Knotenregeln für Leistungen in diesem linearisierten Modell entspricht. Der Vektor der von außen zum System zugeführten Leistungsflüsse lautet $\vec{s}_{\text {bilanz }}=\left[\vec{s}_{\mathrm{WR}}, \vec{s}_{L}\right]^{T}$, wobei die abfließenden Lastflüsse entsprechend negative Werte haben und die Wechselrichterflüsse nach oben und die Lastflüsse nach unten sortiert sind. Damit ergibt sich

$$
0=\mathcal{K} \cdot \vec{s}+\vec{s}_{\text {bilanz }}
$$

\footnotetext{
5 In diesem Fall muss ein weiterer Knoten mit niederimpedanter Verbindung zum Wechselrichter eingebaut werden.

6 Nullen wurden der Übersichtlichkeit halber durch Punkte ersetzt
}

bzw.

$$
-\left[\begin{array}{c}
\vec{s}_{W R} \\
\vec{s}_{L}
\end{array}\right]=\left[\begin{array}{c}
\mathcal{K}_{W R} \\
\mathcal{K}_{N}
\end{array}\right] \cdot \vec{s}
$$

In Gleichung 19 sind die Lastflüsse $\vec{s}_{L}$ bekannt, da sie die Eingangswerte des Systems bilden. Die Wechselrichterleistungen $\vec{s}_{W R}$ sind gesucht, da sie die regelungstechnischen Eingangsvariablen des Wechselrichterteilmodells bilden und deren Ausgangsspannungen bestimmen.

\section{Anmerkung}

Die Vektoren $\vec{s}$ in Gleichung 18 enthalten jeweils zweielementige Komponenten $s_{i}=\left[P_{i}, Q_{i}\right]^{T}$. Die Elemente der Matrix $\mathcal{K}$ enthalten daher diagonalförmige 2x2Submatrizen : $\left[\begin{array}{ll}1 & 0 \\ 0 & 1\end{array}\right]$ anstelle von 1, die Nullmatrix anstelle von 0 und $\left[\begin{array}{cc}-1 & 0 \\ 0 & -1\end{array}\right]$ anstatt von -1 . Zur übersichtlicheren Darstellung wird die einfache Notation von $0,1,-1$ in $\mathcal{K}$ beibehalten.

\subsection{Zweigleistungen}

Die Admittanzwerte $y_{k}=\left[\begin{array}{cc}-B_{k} & G_{k} \\ -G_{k} & -B_{k}\end{array}\right]$ der einzelnen Zweige werden in die blockdiagonale Admittanzmatrix geschrieben, so z.B. für das System aus Abbildung 6:

$$
\mathcal{Y}=\operatorname{diag}\left(y_{1}, y_{2}, y_{3}, y_{4}, y_{5}, y_{6}, y_{7}\right)
$$

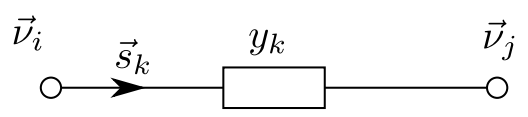

Bild 7: Zweigbeziehung

Mit der negativen transponierten Inzidenzmatrix $-\mathcal{K}^{T}$ können entsprechend Abbildung 7 die Zweigbeziehungen $\vec{s}_{k}=y_{k}\left(\vec{\nu}_{i}-\vec{\nu}_{j}\right)$ ausgerechnet werden:

$$
\vec{s}=-\mathcal{Y} \mathcal{K}^{T} \cdot \vec{\nu}
$$

mit

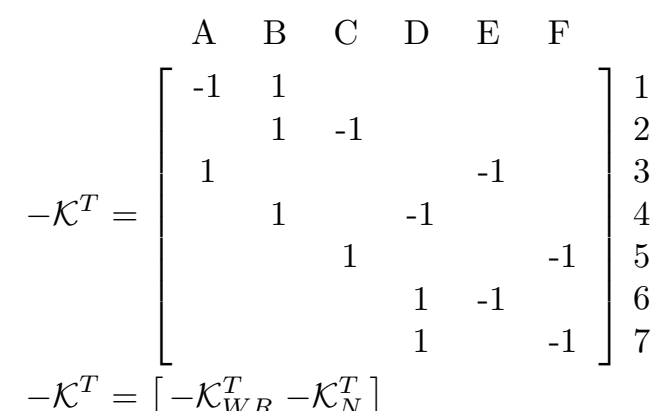

Das Produkt aus Admittanzmatrix und transponierter Inzidenzmatrix kann in eine linksseitige und eine rechtsseitige Teilmatrix aufgespalten werden. Auf Grund der 
Sortierung von $\mathcal{K}^{T}$ befinden sich die Wechselrichterknoten auf der linken Seite und dies passt zu der Sortierung des Spannungsvektors $\vec{\nu}$, in dem die Wechselrichterspannungen oben und die Netzknotenspannungen unten stehen.

$$
\vec{s}=-\mathcal{Y} \mathcal{K}^{T} \cdot \vec{\nu}=\left[-\mathcal{Y} \mathcal{K}_{W R}^{T}-\mathcal{Y} \mathcal{K}_{N}^{T}\right] \cdot\left[\begin{array}{c}
\vec{\nu}_{W R} \\
\vec{\nu}_{N}
\end{array}\right]
$$

In Gleichung 21 können die Wechselrichterspannungen $\vec{\nu}_{W R}$ als bekannt angenommen werden, da diese von den Wechselrichtern vorgegeben werden. Die Knotenspannungen $\vec{\nu}_{N}$ im verbleibenden Netz sind unbekannt.

Wird Gleichung 21 in Gleichung 19 eingesetzt, so erhält man die Beziehung zwischen allen benötigten Größen des Systemmodells:

$$
\begin{aligned}
-\vec{s}_{\text {bilanz }} & =\mathcal{K} \cdot \vec{s} . \\
-\vec{s}_{\text {bilanz }} & =-\mathcal{K} \mathcal{Y} K^{T} \cdot \vec{\nu} \\
\vec{s}_{\text {bilanz }} & =\mathcal{K} \mathcal{Y} K^{T} \cdot \vec{\nu} \\
& =\Upsilon \cdot \vec{\nu}
\end{aligned}
$$

Die Bilanzleistungen $\vec{s}_{\text {bilanz }}$ sind die durch die Wechselrichter zugeführten Leistungen $\vec{s}_{\mathrm{WR}}$ sowie die durch Lasten abfließenden Leistungen $\vec{s}_{\mathrm{L}}$.

$$
\begin{aligned}
& {\left[\begin{array}{c}
\vec{s}_{W R} \\
\vec{s}_{L}
\end{array}\right]=\vec{s}_{\text {bilanz }}} \\
& =\left[\begin{array}{c}
\mathcal{K}_{W R} \\
\mathcal{K}_{N}
\end{array}\right] \mathcal{Y}\left[\mathcal{K}_{W R}^{T} \mathcal{K}_{N}^{T}\right] \cdot \vec{\nu} \\
& =\underbrace{\left[\begin{array}{cc}
\mathcal{K}_{W R} \mathcal{Y} \mathcal{K}_{W R}^{T} & \mathcal{K}_{W R} \mathcal{Y} \mathcal{K}_{N}^{T} \\
\mathcal{K}_{N} \mathcal{Y} \mathcal{K}_{W R}^{T} & \mathcal{K}_{N} \mathcal{Y} \mathcal{K}_{N}^{T}
\end{array}\right]} \cdot\left[\begin{array}{c}
\vec{\nu}_{W R} \\
\vec{\nu}_{N}
\end{array}\right] \\
& =\quad\left[\begin{array}{ll}
\Upsilon_{A} & \Upsilon_{\mathrm{B}} \\
\Upsilon_{\mathrm{C}} & \Upsilon_{\mathrm{D}}
\end{array}\right] \cdot\left[\begin{array}{c}
\vec{\nu}_{W R} \\
\vec{\nu}_{N}
\end{array}\right]
\end{aligned}
$$

- Der Lastvektor $\vec{s}_{L}$ wird von aussen vorgegeben (Systemeingang) und ist bekannt.

- Die Wechselrichterspannungen $\vec{\nu}_{W R}$ ergeben sich über die Wechselrichterleistungen $\vec{s}_{W R}$ aus den Wechselrichtermodellen und können somit auch als bekannt vorausgesetzt werden.

- Die vierteilige Matrix $\left[\begin{array}{ll}\Upsilon_{A} & \Upsilon_{B} \\ \Upsilon_{C} & \Upsilon_{D}\end{array}\right]$ ist die generalisierte Admittanzmatrix.

- Unbekannt sind die Wechselrichterleistungen $\vec{s}_{W R}$ und die Netzspannungen $\vec{\nu}_{N}$.

Gesucht wird deshalb eine Beschreibung der Form

$$
\left[\begin{array}{c}
\vec{s}_{W R} \\
\vec{\nu}_{N}
\end{array}\right]=\left[\begin{array}{cc}
\mathcal{M}_{A} & \mathcal{M}_{B} \\
\mathcal{M}_{C} & \mathcal{M}_{D}
\end{array}\right] \cdot\left[\begin{array}{c}
\vec{\nu}_{W R} \\
\vec{s}_{L}
\end{array}\right]
$$

in der die unbekannten Vektoren $\vec{s}_{W R}$ und $\vec{\nu}_{N}$ mithilfe einer vierteiligen Matrix $\mathcal{M}$ aus den bekannten Vektoren $\vec{\nu}_{W R}$ und $\vec{s}_{L}$ berechnet werden können.

\subsection{Auflösung der Systemgleichungen}

Um die gesuchten unbekannten Vektoren $\vec{s}_{W R}$ und $\vec{\nu}_{N}$ zu finden, wird die Inverse der generalisierten Admittanzmatrix aus Gl. 27 gebildet und auch wieder in vier Teilmatrizen aufgespalten, entsprechend der Anzahl der Wechselrichterknoten und übrig bleibenden Netzknoten.

$$
\begin{aligned}
{\left[\begin{array}{c}
\vec{\nu}_{W R} \\
\vec{\nu}_{N}
\end{array}\right] } & =\Upsilon^{-1} \cdot\left[\begin{array}{c}
\vec{s}_{W R} \\
\vec{s}_{L}
\end{array}\right] \\
& =\mathcal{Z} \cdot\left[\begin{array}{c}
\vec{s}_{W R} \\
\vec{s}_{L}
\end{array}\right] \\
& =\left[\begin{array}{cc}
\mathcal{Z}_{\mathrm{A}} & \mathcal{Z}_{\mathrm{B}} \\
\mathcal{Z}_{\mathrm{C}} & \mathcal{Z}_{\mathrm{D}}
\end{array}\right] \cdot\left[\begin{array}{c}
\vec{s}_{W R} \\
\vec{s}_{L}
\end{array}\right]
\end{aligned}
$$

Die Matrix $\mathcal{Z}$ wird in die Submatrizen $\mathcal{Z}_{\mathrm{A}}, \mathcal{Z}_{\mathrm{B}}, \mathcal{Z}_{\mathrm{C}}$ und $\mathcal{Z}_{\mathrm{D}}$ entsprechend der Anzahl der Wechselrichterknoten und Netzknoten aufgeteilt. Die einzelnen Submatrizen können mithilfe der blockweisen Invertierung des SchurKomplements $\mathcal{S}$ berechnet werden:

$$
\mathcal{S}=\Upsilon_{\mathrm{A}}-\Upsilon_{\mathrm{B}} \Upsilon_{\mathrm{D}}^{-1} \Upsilon_{\mathrm{C}}
$$

Es müssen nur die Matrix $\Upsilon_{\mathrm{D}}$ und das Schurkomplement $\mathcal{S}$ invertiert werden und alle anderen Teilinversen können daraus berechnet werden:

$$
\begin{aligned}
\mathcal{Z}_{\mathrm{A}} & =\mathcal{S}^{-1} \\
\mathcal{Z}_{\mathrm{B}} & =-\mathcal{S}^{-1} \Upsilon_{\mathrm{B}} \Upsilon_{\mathrm{D}}^{-1} \\
\mathcal{Z}_{\mathrm{C}} & =-\Upsilon_{\mathrm{D}}^{-1} \Upsilon_{\mathrm{C}} \mathcal{S}^{-1} \\
\mathcal{Z}_{\mathrm{D}} & =\Upsilon_{\mathrm{D}}^{-1}+\Upsilon_{\mathrm{D}}^{-1} \Upsilon_{\mathrm{C}} \mathcal{S}^{-1} \Upsilon_{\mathrm{B}} \Upsilon_{\mathrm{D}}^{-1}
\end{aligned}
$$

Das Schurkomplement kann sowohl über $\Upsilon_{\mathrm{D}}$, als auch über $\Upsilon_{\mathrm{A}}$ gebildet werden. Für eine numerische Lösung, z.B. in MATLAB hat die Auswahl Einfluss auf die numerische Güte des Invertierungsalgorithmus, die z.B. durch eine Konditionierungszahl von $\mathcal{S}$ ausgedrückt wird.

Mit den Gleichungen 27 und 31 können die Submatrizen aus Gl. 28 berechnet werden. Der untere Teil der Gleichung 31 lautet

$$
\vec{\nu}_{N}=\mathcal{Z}_{\mathrm{C}} \cdot \vec{s}_{W R}+\mathcal{Z}_{\mathrm{D}} \cdot \vec{s}_{L}
$$

Der obere Teil der Gleichung 27 lautet

$$
\vec{s}_{W R}=\Upsilon_{\mathrm{A}} \cdot \vec{\nu}_{W R}+\Upsilon_{\mathrm{B}} \cdot \vec{\nu}_{N}
$$

Setzt man Gleichung 34 für $\vec{s}_{W R}$ in Gl. 33 ein, so erhält man

$$
\vec{\nu}_{N}=\mathcal{Z}_{\mathrm{C}} \cdot\left(\Upsilon_{\mathrm{A}} \vec{\nu}_{W R}+\Upsilon_{\mathrm{B}} \vec{\nu}_{N}\right)+\mathcal{Z}_{\mathrm{D}} \cdot \vec{s}_{L}
$$

und nach Umstellung und Auflösung nach $\vec{\nu}_{N}$

$$
\begin{array}{r}
\vec{\nu}_{N}=\left(\mathcal{I}_{l}-\mathcal{Z}_{\mathrm{C}} \Upsilon_{\mathrm{B}}\right)^{-1} \mathcal{Z}_{\mathrm{C}} \Upsilon_{\mathrm{A}} \cdot \vec{\nu}_{W R} \\
+\left(\mathcal{I}_{l}-\mathcal{Z}_{\mathrm{C}} \Upsilon_{\mathrm{B}}\right)^{-1} \mathcal{Z}_{\mathrm{D}} \cdot \vec{s}_{L}
\end{array}
$$


mit $\mathcal{I}_{l}$ als Einheitsmatrix der Dimension $l$, mit $l=n-w$ gleich der Anzahl der Lastknoten. Durch Vergleich mit dem unteren Teil der Gleichung 28 ergibt sich

$$
\begin{aligned}
& \mathcal{M}_{C}=\left(\mathcal{I}_{l}-\mathcal{Z}_{\mathrm{C}} \Upsilon_{\mathrm{B}}\right)^{-1} \mathcal{Z}_{\mathrm{C}} \Upsilon_{\mathrm{A}} \\
& \mathcal{M}_{D}=\left(\mathcal{I}_{l}-\mathcal{Z}_{\mathrm{C}} \Upsilon_{\mathrm{B}}\right)^{-1} \mathcal{Z}_{\mathrm{D}}
\end{aligned}
$$

Einsetzen von Gleichung 36 in Gl. 34 ergibt

$$
\begin{aligned}
\vec{s}_{W R} & =\Upsilon_{\mathrm{A}} \cdot \vec{\nu}_{W R}+\Upsilon_{\mathrm{B}} \cdot\left(\mathcal{M}_{C} \cdot \vec{\nu}_{W R}+\mathcal{M}_{D} \cdot \vec{s}_{L}\right) \\
& =\left(\Upsilon_{\mathrm{A}}+\Upsilon_{\mathrm{B}} \mathcal{M}_{C}\right) \cdot \vec{\nu}_{W R}+\Upsilon_{\mathrm{B}} \mathcal{M}_{D} \cdot \vec{s}_{L}
\end{aligned}
$$

Durch Vergleich mit dem oberen Teil der Gleichung 28 ergibt sich

$$
\begin{aligned}
& \mathcal{M}_{A}=\Upsilon_{\mathrm{A}}+\Upsilon_{\mathrm{B}} \mathcal{M}_{C} \\
& \mathcal{M}_{B}=\Upsilon_{\mathrm{B}} \mathcal{M}_{D}
\end{aligned}
$$

bzw.

$$
\begin{aligned}
& \mathcal{M}_{A}=\Upsilon_{\mathrm{A}}+\Upsilon_{\mathrm{B}}\left(\mathcal{I}_{l}-\mathcal{Z}_{\mathrm{C}} \Upsilon_{\mathrm{B}}\right)^{-1} \mathcal{Z}_{\mathrm{C}} \Upsilon_{\mathrm{A}} \\
& \mathcal{M}_{B}=\Upsilon_{\mathrm{B}}\left(\mathcal{I}_{l}-\mathcal{Z}_{\mathrm{C}} \Upsilon_{\mathrm{B}}\right)^{-1} \mathcal{Z}_{\mathrm{D}}
\end{aligned}
$$

Damit ist das System vollständig bestimmt. Die Matrizen $\mathcal{M}_{A}, \mathcal{M}_{B}, \mathcal{M}_{C}$ und $\mathcal{M}_{D}$ können aus der Inzidenzmatrix $\mathcal{K}$ bzw. aus deren Teilmatrizen $\mathcal{K}_{W R}$ und $\mathcal{K}_{N}$, aus der Admittanzmatrix $\mathcal{Y}$, sowie aus der Inversen $\mathcal{Z}=\left(\mathcal{K} \mathcal{Y} K^{T}\right)^{-1}$ bzw. deren Teilmatrizen berechnet werden.

Zusammenfassend ergeben sich aus den Gleichungen 37 bis 40:

- $\mathcal{M}_{A}=\Upsilon_{\mathrm{A}}+\Upsilon_{\mathrm{B}}\left(\mathcal{I}_{l}-\mathcal{Z}_{\mathrm{C}} \Upsilon_{\mathrm{B}}\right)^{-1} \mathcal{Z}_{\mathrm{C}} \Upsilon_{\mathrm{A}}$

- $\mathcal{M}_{B}=\Upsilon_{\mathrm{B}}\left(\mathcal{I}_{l}-\mathcal{Z}_{\mathrm{C}} \Upsilon_{\mathrm{B}}\right)^{-1} \mathcal{Z}_{\mathrm{D}}$

- $\mathcal{M}_{C}=\left(\mathcal{I}_{l}-\mathcal{Z}_{\mathrm{C}} \Upsilon_{\mathrm{B}}\right)^{-1} \mathcal{Z}_{\mathrm{C}} \Upsilon_{\mathrm{A}}$

- $\mathcal{M}_{D}=\left(\mathcal{I}_{l}-\mathcal{Z}_{\mathrm{C}} \Upsilon_{\mathrm{B}}\right)^{-1} \mathcal{Z}_{\mathrm{D}}$

Das Gesamtmodell der Zustandsraumdarstellung kann entsprechend der Abbildung 8 modelliert werden und ergibt sich direkt aus Gleichung 28.

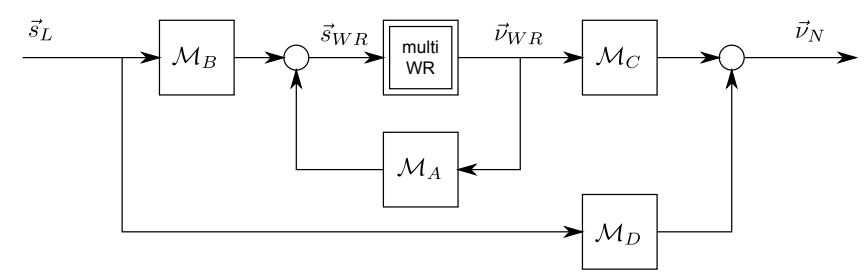

Bild 8: Netzmodell

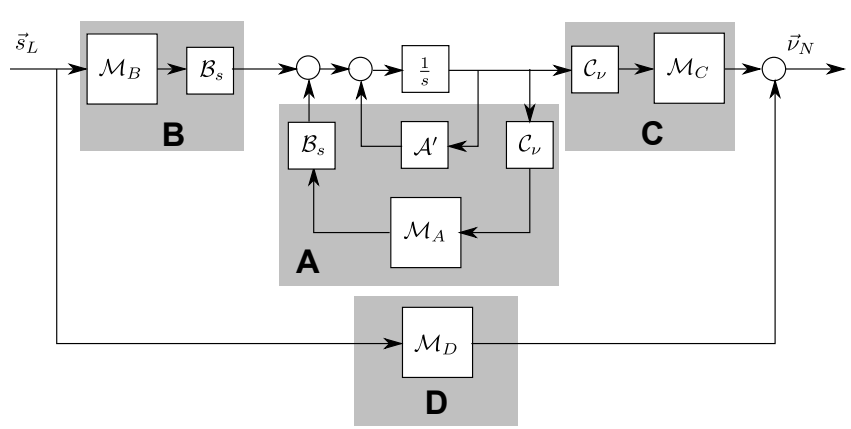

Bild 9: Zusammenfassung zu globalen Systemmatrizen

\subsection{Regelungstechnisches Gesamtmodell}

Das Multi-Wechselrichtermodell aus 3.2 (siehe auch Abb. 4) kann für die weitere regelungstechnische Beschreibung in das Netzmodell integriert werden.

Dabei wird die Matrix $\mathcal{B}_{s}$ nach vorne über die Summationsstelle gezogen und die Matrix $\mathcal{C}_{\nu}$ nach hinten über den Abzweig geschoben.

Die Matrizen können dann, wie in Abbildung 9 dargestellt, zu den globalen Systemmatrizen zusammengefasst werden:

$$
\begin{aligned}
\mathcal{A} & =\mathcal{A}^{\prime}+\mathcal{B}_{s} \mathcal{M}_{A} \mathcal{C}_{\nu} \\
\mathcal{B} & =\mathcal{B}_{s} \mathcal{M}_{B} \\
\mathcal{C} & =\mathcal{M}_{C} \mathcal{C}_{\nu} \\
\mathcal{D} & =\mathcal{M}_{D}
\end{aligned}
$$

Daraus kann die Übertragungsfunktion im Frequenzbereich bestimmt werden mit

$$
G(s)=\mathcal{C}\left(s \mathcal{I}_{n}-\mathcal{A}\right)^{-1} \mathcal{B}+\mathcal{D}
$$

Mit diesem linearisierten Modell können die Untersuchungen zur Stabilität des Gesamtsystems und der Entwurf für eine robuste Netzregelung durchgeführt werden.

- Unterschiedliche Netzstrukturen werden durch die Inzidenzmatrix $\mathcal{K}$ beschrieben und alle Strukturen werden gleichförmig berechnet. Liegt die Inzidenzmatrix eines Netzes vor, kann das Modell daraus z.B. mit MATLAB leicht generiert werden.

- Über die Admittanzmatrix können verschiedene Spannungsebenen und deren unterschiedliche Leitungscharakteristika (ohmsch, ohmsch-induktiv, induktiv) untersucht werden.

- Beliebig viele Wechselrichter können im Modell integriert werden, ohne jeweils von vorne die Differentialgleichungen aufstellen zu müssen.

- Unterschiedliche Wechselrichterdynamiken können durch Anpassung des WR-Modells berücksichtigt werden. Zustandsraumdarstellungen mit beliebiger dynamischer Genauigkeit und Systemordnung 
können für die Wechselrichter eingesetzt werden. Dabei ist es möglich unterschiedliche WechselrichterKonfigurationen innerhalb einer Netzstruktur zu untersuchen.

- Das entwickelte Modell ist sowohl auf reine Inselnetze anwendbar, als auch auf Teilnetze, die an einer langen Leitung am starren Netz angeschlossen sind. Dazu kann z.B. ein Wechselrichter an einem hochohmigen Leitungszweig als starres Netz modelliert werden, indem er eine große Zeitkonstante und sehr große Nennleistung erhält, oder als Wechselrichter mit voreingestellter, nicht steuerbarer Spannung modelliert wird.

\section{Dynamik-Analyse und Anwendung}

An kurzen Beispielen soll die Anwendbarkeit des Modells zur Analyse der Netzdynamik demonstriert werden.

Dazu wird ein Gitternetz betrachtet, bei dem in jedem Kreuzungspunkt ein Wechselrichter sitzt. Jeweils zwischen den Wechselrichtern sind Lastknoten angenommen. Das modellierte Netz zeigt Abbildung 10, in der alle Wechselrichterknoten als Kreis, und alle Lastknoten als Dreieck dargestellt sind.

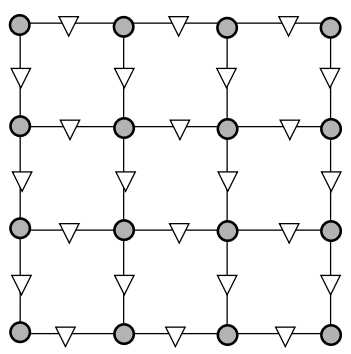

Bild 10: Gitterstruktur

Modelliert wurde ein Niederspannungsnetz mit 400V Nennspannung, mit jeweils gleicher Leitungssegmentlänge $L=500 \mathrm{~m}$ und den spezifischen Leitungsimpedanzen ${ }^{7} R^{\prime}=0.642 \frac{\Omega}{\mathrm{km}}$ und $X^{\prime}=0.083 \frac{\Omega}{\mathrm{km}}$.

Die Wechselrichter wurden jeweils mit dem gleichen Modell 5. Ordnung modelliert und mit exakt gleichen Werten im Modell implementiert:

- Frequenzstatik $k_{P}=2 \% / P_{n}$

- Spannungsstatik $k_{Q}=4 \% / Q_{n}$

- Zeitkonstante $T_{\mathrm{WR}}=100 \mathrm{~ms}$

In [14] wurde ein erstes Pol-Nullstellen-Bild eines kleinen Inselnetzes vorgestellt. Im folgenden wird auch das Pol-Nullstellen-Diagramm zur Analyse herangezogen.

Beispielhaft soll der Einfluss der WechselrichterNennleistung auf die Systemstabilität betrachtet werden. Wird ein Netz mit immer kleinerer WechselrichterNennleistung modelliert, so führt dies ab einem gewissen

7 Werte für $R^{\prime}$ und $X^{\prime}$ aus [4, (Tabelle S.99)]
Punkt zur Instabilität. Zur Visualisierung dieses Effekts wurden mehrere Modelle des o.g. Netzes berechnet. Dabei sind die Wechselrichter sind immer identisch und in jeder Modellvariation werden sie mit einer kleineren Nennleistung modelliert. Die Pole aller Modelle werden im selben PN-Diagramm von Abbildung 11 dargestellt, wobei für jedes Modell, d.h. für jede Nennleistung, eine andere Farbe verwendet wird. Der Farbverlauf zeigt die Veränderung der Position der Polregionen und die korrespondierende Nennleistung ist auf dem rechten Farbbalken wiedergegeben.

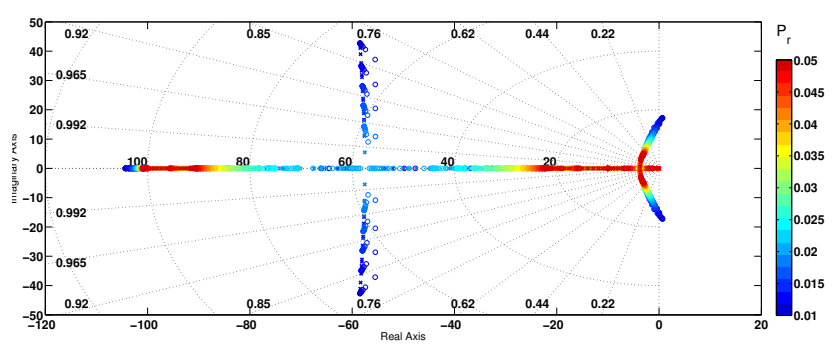

Bild 11: Polregionen bei Variation der Nennleistung $P_{\mathrm{n}}$

Nahe der imaginären Achse bilden sich konjugiertkomplexe, dominante Polpaare mit sehr schwacher Dämpfung, die mit sinkender Nennleistung in die rechte Halbebene wandern. Abbildung 12 zeigt die rechte Polregion im Detail.

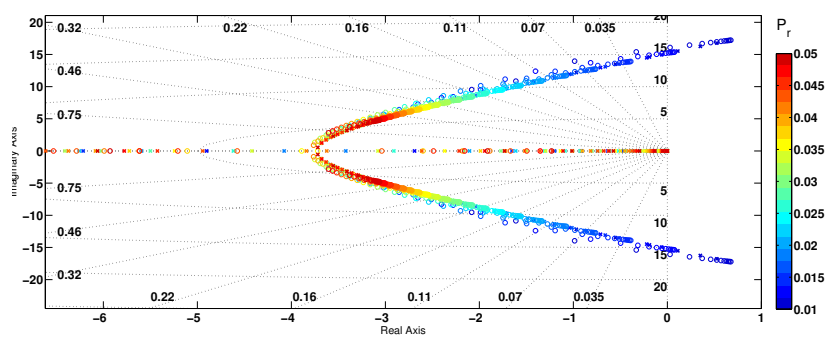

Bild 12: Instabile Pole für kleine $P_{\mathrm{n}}$ in Polregion $\mathrm{C}$

Die physikalische Ursache der nennleistungsabhängigen Instabilität liegt in der Tatsache, dass im Statikregler des Wechselrichters bei der Berechnung der Einspeisefrequenz $f_{\text {VsI }}$ die Steigung der Statik auf die Nennleistung $P_{n}$ bezogen wird, wie Gleichung 46 zeigt.

$$
f_{\mathrm{VSI}}=\frac{2 \%}{P_{n}} P_{\mathrm{WR}}+f_{0}
$$

Wird in der Bestimmungsgleichung die Nennleistung $P_{\mathrm{n}}$ immer kleiner, so wird die Reaktion auf eine anliegende Klemmenleistung $P_{\mathrm{WR}}$ in Form der Frequenzabweichung von der Nennfrequenz immer stärker. Starke Frequenzschwankungen im System führen zu starken Reaktionen der anderen Wechselrichter im Netz, zu starken 
Leistungsschwankungen und letztlich zur formalen Instabilität. Die Reaktion eines instabilen Netzes auf einen Lastsprung ist in der Simulation von Abbildung 13 dargestellt.
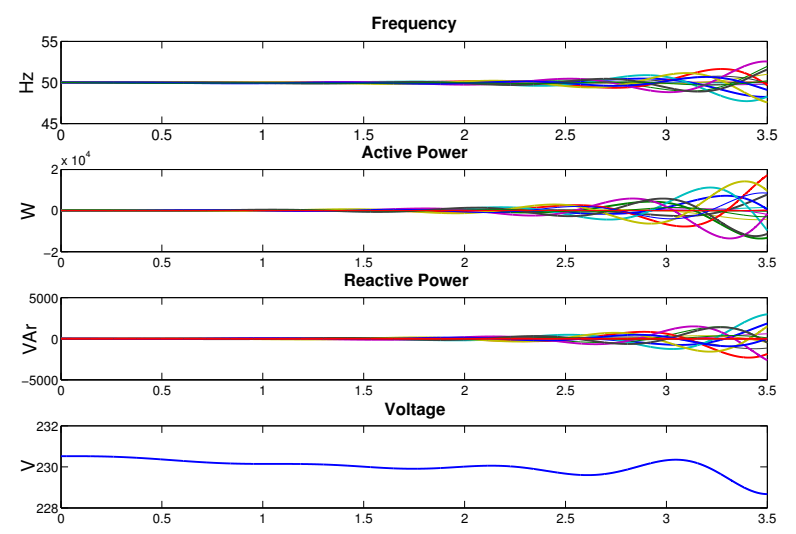

Bild 13: Instabile Reaktion von 16 Wechselrichtern auf einen Lastsprung

Diese leistungsbezogene Instabilität hängt ebenfalls mit der Zeitkonstanten $T_{\text {WR }}$ zusammen. Da kleine Nennleistungen $\mathrm{zu}$ relativ starken Frequenzschwankungen führen, kann das System stabilisiert werden, indem die anderen Wechselrichter schneller darauf reagieren, d.h. wenn die Zeitkonstante $T_{\mathrm{WR}}$ der Wechselrichter verkleinert wird. Den Effekt zeigt Abbildung 14.

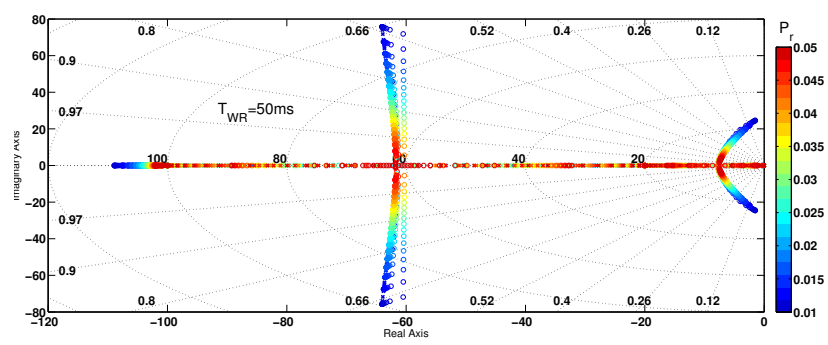

Bild 14: Stabilisierung durch Linksverschiebung bei kleinen Zeitkonstanten

Für die gleiche Schwankungsbreite der Nennleistung $P_{\mathrm{n}}$ wie in Abb. 11 wurde in Abb. 14 für alle Wechselrichter die kleinere Zeitkonstante $T_{\mathrm{WR}}=50 \mathrm{~ms}$ verwendet, was die Polregionen nach links und wieder vollständig in die linke Halbebene schiebt, hingegen auf Kosten der Dämpfung, wie ein Vergleich der Abbildungen 11 und 14 zeigt.

Weitergehende Betrachtungen über den Einfluss anderer Modellparameter auf die Systemdynamik auf Basis des vorgestellten Modells finden sich in [7] und [8]. Analysiert werden dort u.a. der Einfluss der Anzahl der Wechselrichter im Netz, die erhöhte dynamische Kopplung durch kurze Leitungen oder der Einfluss der Dämpfung im Regelkreis. Auch die Erhöhung der Integrationsdichte von Wechselrichtern im Netz durch die in [9] vorgestellte P/Q-Rotation kann dort nachgewiesen werden.

\section{Zusammenfassung und Ausblick}

Der Artikel stellt die detaillierte Entwicklung eines regelungstechnischen Modells für wechselrichtergeführte Inselnetze vor. Der Zugang liegt in der Aufteilung des Netzes in solche Knoten, an denen Wechselrichter angeschlossen sind, und alle anderen Knoten, an denen Lasten abfließen können. Ausgehend von der Zustandsraumdarstellung eines einzelnen Wechselrichters, wird ein Multi-Wechselrichtermodell durch blockdiagonale Aggregation erstellt. Für das passive Netz werden mithilfe der Knoteninzidenzmatrix die linearisierten Leistungsflussgleichungen aufgestellt und nach den unbekannten Größen aufgelöst. Ein Teil der Unbekannten werden mit dem Multi-Wechselrichtermodell berechnet. Das Zusammenfügen von dynamischem Wechselrichtermodell und passivem Netzmodell ergibt eine Mehrgrößen-Zustandsraumdarstellung des gesamten Netzes. Auf Grund des strukturierten Zugangs können auch Modelle großer Netze und mit vielen Wechselrichtern effizient bestimmt werden, ohne manuelle Erstellung aller Differentialgleichungen. Die Anwendbarkeit des Modells zur Stabilitätsanalyse wurde anhand eines Beispiels präsentiert.

Im vorgestellten Beispiel wurde ein genereller Ansatz verwendet, in dem alle Wechselrichter identisch modelliert wurden. Für ein Beispiel mit einer höheren Anzahl Wechselrichter sollten im nächsten Schritt ihre Parameter stochastisch verteilt sein um eine realistische Bandbreit abzudecken. Als weitere Möglichkeiten dieses Modells bietet sich die Analyse eines real existierenden Teilbereichs eines Versorgungsnetzes an, der für den Inselnetzbetrieb untersucht werden soll.

Dieses Projekt wird durchgeführt am Interdisciplinary Center for Security, Reliability and Trust (SnT) an der Universität Luxemburg und wird gefördert durch den Fonds National de la Recherce (FNR) Luxemburg unter der Projektnummer PHD-09-183 


\section{Literaturverzeichnis}

[1] Ongun Alsaç Brian Stott, Jorge Jardim. Dc power flow revisited. IEEE Transactions on Power Systems, 24(3):1290-1300, August 2009.

[2] Pascal Van Hentenryck Carleton Coffrin. Approximating line losses and apparent power in ac power flow linearizations. Power and Energy Society General Meeting, 2012 IEEE, pages 1-8, July 2012.

[3] Luigi Iannelli Carmine Di Pietro, Francesco Vasca. Decentralized synchronization of parallel inverters for train auxiliaries. Electrical Systems for Aircraft, Railway and Ship Propulsion (ESARS), pages 1-6, October 2010.

[4] Alfred Engler. Regelung von Batteriestromrichtern in modularen und erweiterbaren Inselnetzen. $\mathrm{PhD}$ thesis, Universität Gesamthochschule Kassel, May 2001.

[5] Jürgen Sachau et.al. Control of parallel working power units in expandable grids. In Proceedings of the 14th European PV Solar Energy Conference and Exhibition, Barcelona, 1997.

[6] Ross T. Guttromson. Modeling distributed energy resource dynamics on the transmission system. IEEE Transactions on Power Systems, 17(4):11481153, November 2002.

[7] Markus Jostock. Stabilität wechselrichtergeführter Inselnetze. Dissertation, Universität Luxemburg, Campus Kirchberg, 6, rue Coudenhove-Kalergi, 1359 Luxembourg, Juni 2013.

[8] Markus Jostock, Jürgen Sachau, Jean-Régis HadjiMinaglou, and Christian Tuttas. Structured analysis of arbitrary island grids. Proceedings of 4 th European Conference on Innovative Smart Grid Technologies, 2013.

[9] R. Belmans K. DeBrabandere, B. Bolsens. A voltage and frequency droop control method for parallel inverters. In 35th Annual IEEE Power Electronics Specialists Conference, pages 2501-2507, 2004.

[10] W. Kleinkauf and J. Sachau. EUREC agency's mega-hybrid project: Electric system technology for renewable energy stand alone applications in the frame of the JOULE II programme. EUREC Newsletter, 1, 1994.

[11] T.C. Green N. Kroutikova, C.A. HernandezAramburo. State-space model of grid connected inverters under current control mode. IET Electrical Power Applications, 1(3):329-338, 2007.

[12] Oleg Osika. Stability of MicroGrids with High Share of Inverter-dominated and Decentralised Sources. Phd thesis, University of Kassel, 2005.

[13] J. Ossana, H. Graner, and F. Hoffmann. Frequenz und Leistungssteuerung (Netzkennliniensteuerung) von Netzverbänden. German Patent DRP-634025, 1931.
[14] Arindam Ghosh Ritwik Majumber, Balarko Chaudhuri. Improvement of stability and load sharing in an autonomous microgrid using supplementary droop control. IEEE Transactions on Power Systems, 25(2):796-808, May 2010.

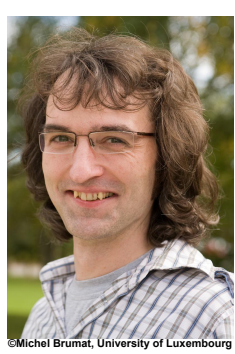

Dipl.-Ing. Markus Jostock promoviert am Interdisciplinary Center for Security, Reliability and Trust (SnT) an der Universität Luxemburg über Stabilität wechselrichtergeführter Inselnetze.

Adresse: Universität Luxemburg, Fakultät für Naturwissenschaften, Technologie und Kommunikation, 6 rue CoudenhoveKalergi, L-1359 Luxemburg, E-Mail: markus.jostock@uni.lu

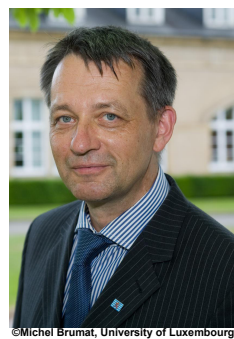

Prof. Dr.-Ing. Jürgen Sachau Jürgen Sachau ist Professor für System- und Regelungstechnologie der Universität Luxemburg und leitet den Forschungsbereich Zuverlässige Vernetzte Energiesysteme im Interdisciplinary Center for Reliability, Security und Trust sowie das Netpower Lab im internationalen Laborverbund DERlab zur Integration dezentraler, erneuerbarer Energiequellen in die Energieversorgungsnetze. Sein Forschungsinteresse für rekursive Strukturen und zuverlässige Dynamiken konzentriert sich auf die TechnologieEntwicklung für grossflächige, nachhaltige Energiesysteme auf dem Weg zur solaren Vollversorgung.

Adresse: Universität Luxemburg s.o.

E-Mail: juergen.sachau@uni.lu

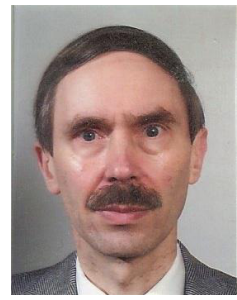

Dr.-Ing. habil. Christian Tuttas ist Privatdozent an der Universität Kaiserslautern am Lehrstuhl für Regelungssysteme. Sein Forschungsgebiet sind elektrische Energieversorgungsnetze und umfasst die Modellbildung, Regelung und Simulation leistungselektronischer Betriebsmittel (FACTS) im Netzverbund.

Adresse: Universität Kaiserslautern, Fachbereich Elektrotechnik, Lehrstuhl für Regelungssysteme, Postfach 3049, 67653 Kaiserslautern E-Mail: tuttas@eit.uni-kl.de 